\title{
Travail pratique de mesure optique 3D utilisant un montage expérimental simple
}

\author{
Daniel Mathieu ${ }^{\mathrm{a}}$ \\ École Nationale Supérieure d'Ingénieur du Sud Alsace (ENSISA), Laboratoire de Physique et Mécanique Textiles \\ (LPMT) CNRS UMR 7189, 11 rue Alfred Werner, 68093 Mulhouse Cedex, France
}

Reçu le 28 août 2008, accepté le 9 avril 2009

\begin{abstract}
Résumé - La rétro-conception géométrique est un processus permettant l'obtention d'un modèle numérique à partir d'un objet réel préexistant. Le processus de prise de mesures est l'étape initiale conditionnant cette démarche. Plusieurs solutions commerciales performantes existent, mais sont trop souvent des boîtes noires pour l'utilisateur. Les limites inhérentes aux principes physiques utilisés ne sont pas directement perceptibles. Dans le cadre de travaux pratiques de rétro-conception, les étudiants expérimentent la mesure 3D d'un élément de carrosserie avec un montage simple qui se compose d'un vidéoprojecteur et d'un appareil photo numérique. La méthode testée est celle de la lumière structurée avec codage en binaire réfléchi de la position des franges. À partir de la disposition spatiale du dispositif expérimental et des prises de vue, les étudiants doivent analyser le contenu 3D des images à l'aide de logiciels génériques non dédiés. Ceci leur permet d'estimer les sources d'erreurs expérimentales ou de traitement des images. L'étape ultime est l'intégration des données numérisées dans une solution CAO où une comparaison avec un modèle numérique est possible.
\end{abstract}

Mots clés : Travail pratique / rétro-conception / mesure optique/ lumière structurée / traitement d'images / erreurs de mesure

\begin{abstract}
Practical work of optical 3D measurement with a simple experimental device. The geometric reverse engineering is a process for obtaining a CAD model from a pre-existing real object. The measuring process is the initial stage which controls this method. Several successful commercial solutions exist but they are in many cases like "black boxes" for the user. The inherent limitations of the physical principles used are not directly observable. Under practical work of reverse engineering, students are experimenting with 3D measurements of an objet with a simple device. This device consists of a video projector and a digital camera. The tested method is the structured light where patterns are used to do a reflected binary encoding. So it is easy to identify the position of fringes. From the spatial positioning of the experimental device elements and the images, the students have to find out the 3D geometry. For this, they use generic and non-dedicated softwares. This allows them to estimate the experimental and image processing errors. The final step is the integration of digital data in a CAD software where a comparison with a digital model is possible.
\end{abstract}

Key words: Work practice / reverse-engineering / optical measurement / structured light / image processing / measurement errors

\section{Introduction}

Le processus de conception permet à partir d'un cahier des charges d'aboutir, via l'étape de fabrication, à un objet réel issu d'une modélisation informatique. La

\footnotetext{
a Auteur pour correspondance : daniel.mathieu@uha.fr
}

rétro-conception géométrique est le processus inverse de la démarche classique. Contrairement à l'habitude, une certaine représentation physique de l'objet existe alors qu'aucun modèle numérique n'est encore défini. Or, ce modèle informatique est un préalable indispensable au processus de fabrication par des machines d'usinage à commande numériques ou de prototypage par ajout de 
matière. La raison de cette inversion est à chercher au niveau de l'origine de la géométrie physique disponible. Ainsi, le modèle peut être une pièce ancienne qu'il faut remplacer. Dans d'autres cas la géométrie provient d'un modèle fait à la main (maquette, œuvre d'art) ou bien encore d'un élément biologique auquel le dispositif conçu doit s'adapter au mieux (cas des prothèses).

De quel type de modèle numérique l'industriel a-til besoin? À part le vieux modèle filaire qui n'a plus cours, seuls deux types de modèles numériques existent. Le premier est le modèle à facettes décrivant aussi bien des « surfaces » que des volumes. Les facettes sont triangulaires et définie par leurs sommets et un vecteur normal donnant l'orientation à la facette (de l'intérieur, s'il existe, vers l'extérieur du modèle). L'avantage de cette représentation est qu'elle peut résulter plus ou moins directement du nuage de points obtenu lors de la mesure 3D. Par contre cette représentation est discontinue par nature et cette propriété peut se voir sur la pièce usinée. Le second est le modèle paramétrique à base de volumes ou des surfaces interconnectées (B-rep). Les modèles surfaciques sont composés de carreaux surfaciques définis par des familles de courbes polynomiales paramétriques décrivant aussi bien les frontières que tout point interne. L'avantage de cette représentation est de permettre le contrôle des conditions de continuité dans et entre les patchs à partir d'un nombre limité de points de contrôle. Ainsi le modèle est plus léger en taille mémoire et plus « précis ». Néanmoins, il faut faire appel à des procédures d'interpolation pour ajuster chaque élément surfacique à une zone du nuage de points. Cette procédure peut être également appliquée pour définir des surfaces plus classiques comme des cylindres, des plans, des sphères, etc. Comment alors faire la prise de mesure 3D afin de recueillir un nuage de points le plus représentatif possible? Les méthodes existantes peuvent être mécaniques ou optiques avec ou non destruction de l'objet. Le choix de la méthode résulte de la conjonction entre leurs limitations, les caractéristiques de l'objet à numériser et le but recherché entre autres choses.

Afin que les élèves ingénieurs en mécanique de l'ENSISA (École Nationale Supérieure d'Ingénieurs du Sud Alsace) connaissent les méthodes de mesure et les étapes de traitement nécessaires au processus de rétroconception un module de cours de travaux pratiques de rétro-conception existe en troisième année. L'une des activités proposée est la mesure 3D par une méthode optique d'un élément de carrosserie de voiture. Dans un premier temps les étudiants découvrent le matériel expérimental constitué d'éléments du commerce comme un vidéo projecteur, un appareil photo numérique et un ordinateur. À partir des images obtenues de l'objet à numériser ils doivent faire les différentes étapes du traitement par des procédures qu'ils écrivent eux-mêmes. Le but de cette expérience est d'une part de démystifier la mesure 3D par la maîtrise du début à la fin du processus de mesure et d'autre part de permettre aux étudiants d'identifier les sources d'erreurs expérimentales (précision de la position des éléments du montage, zones cachées ou de luminosité non homogène...) et de traitement (déformation optique de l'image, mauvaise identification de la frange...).

\section{La méthode de mesure optique}

Une photographie est une projection du monde tridimensionnel sur une surface plane. L'analyse de la photographie ne peut permettre directement la reconstruction du relief. Certaines hypothèses ou conditions expérimentales rendent l'opération possible. Par exemple l'intensité lumineuse peut dépendre du relief de l'objet. Pour des objets prismatiques la détection des contours peut, avec la connaissance des conditions géométriques de prise de vue, permettre également une reconstruction $3 \mathrm{D}$. Une autre possibilité est de rajouter volontairement une information supplémentaire comme des motifs lumineux. Le relief de l'objet va alors interagir avec ces motifs et coder la géométrie 3D à définir. Ceci nécessite toutefois la connaissance des propriétés de l'appareil photographique (la longueur focale, la déformation de l'image par le système optique) et de la disposition relative entre le dispositif de prise de mesure et l'objet à mesurer (orientation de l'axe optique, distances entre l'émetteur et le récepteur...). L'établissement de la matrice de passage (opération de calibrage) entre les deux repères est une opération indispensable pour obtenir des dimensions fiables.

La méthode optique retenue pour ce travail pratique fait partie des méthodes classiques et consiste à projeter suivant une direction connu un réseau de franges lumineuses. Ce réseau peut être décrit par une fonction périodique de type sinusoïdale ou encore de type créneau. Puis le motif projeté est observé dans une direction décalée angulairement par rapport à la direction de projection. Il présente alors des déformations et des zones manquantes. Les déformations résultent du décalage dans la direction normale aux franges causé par le relief de l'objet. Ce principe est illustré sur la figure 1. La profondeur suivant la direction $Z$ est convertie en un déplacement le long de la direction $x$ sur le capteur de l'appareil photographique. Ainsi comme pour la plus grande partie des méthodes optiques de métrologie, c'est la phase du motif périodique qui code l'information dimensionnelle. C'est donc ce paramètre qu'il convient de mesurer avec précision en chaque point de la zone d'intérêt.

Plusieurs méthodes existent pour la mesure de ce paramètre comme le décalage de phase qui nécessite la projection de motifs dont l'intensité lumineuse est une sinusoïde parfaite avec des déphasages choisis. Par combinaison linéaire des images, une cartographie périodique de la phase est obtenue. Cette cartographie nécessite alors une étape supplémentaire de dépliement pour avoir une phase variant de 0 à $2 \pi N$ où $N$ est le nombre de motifs utiles. De part la relative complexité de traitement et les nombreuses variantes de cette approche, une autre méthode plus directe, toutefois moins précise spatialement, est alors proposée aux étudiants : le codage en binaire réfléchi. Néanmoins, avant cette proposition, ils 


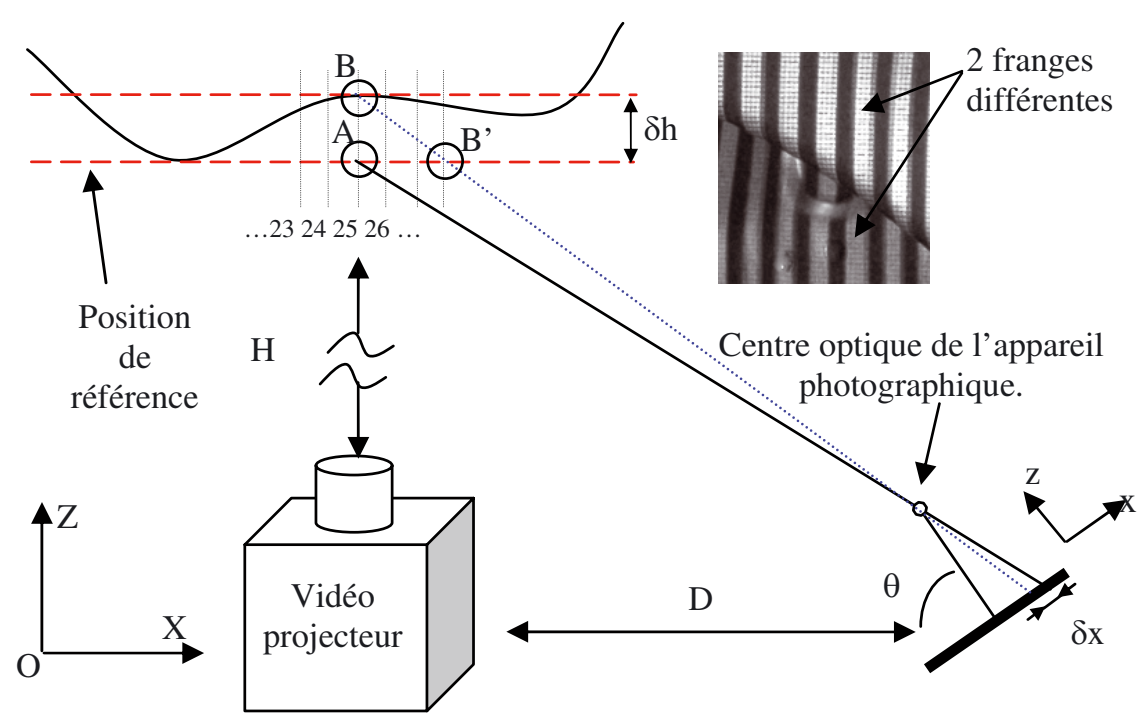

Fig. 1. Schéma du dispositif de mesure par lumière structurée. Ce schéma illustre la nécessité d'une mesure fiable de la phase.

seront amenés à tester la méthode naïve de comptage des franges par une programmation. Après la définition de la méthode de mesure d'autres difficultés expérimentales seront étudiées comme les conditions d'éclairage, la précision de positionnement des éléments du dispositif de mesure et le choix des paramètres d'extraction des données 3D des clichés photographiques.

\section{Choix d'une méthode de mesure de la phase}

La première difficulté à surmonter est de choisir une bonne méthode de mesure de la phase des motifs périodiques. L'étudiant va d'abord étudier l'approche naïve consistant à mesurer la phase de proche en proche en parcourant ligne par ligne l'image. L'enseignant lui propose alors de numéroter chaque frange par le code Gray afin de s'affranchir d'un certain nombre d'écueils détecté avec la première approche.

\subsection{Analyse d'une méthode naïve}

La première idée pour mesurer la phase du motif photographié est de suivre de proche en proche les franges suivant chaque ligne de l'image. Pour ce faire il est possible de tracer l'évolution de l'intensité lumineuse d'une extrémité à l'autre de l'image. Puis, par une procédure de détection des pics de luminosité il est possible de définir la phase du motif et la position correspondante dans l'image. Cette méthode naïve souvent proposée par l'étudiant est mise à mal par au moins deux phénomènes.

Le premier est que le signal à analyser présente des biais liés à des modifications de luminosité et de contraste à la surface de la paroi solide comme le montre la figure 2. Il apparaît clairement que l'identification de la

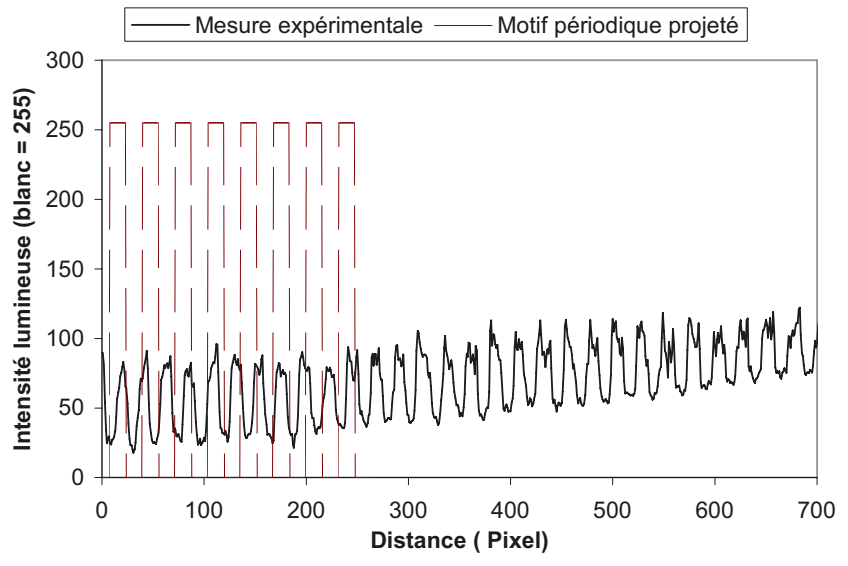

Fig. 2. Comparaison entre le motif théorique et celui mesuré. L'évolution de la luminosité en fonction de la position montre la présence des biais expérimentaux.

phase du signal expérimental nécessite l'utilisation de procédures insensible aux fluctuations du signal. Mais ceci sera réalisé au prix d'une perte de précision spatiale. Ce problème sensibilise l'étudiant à la difficulté des mesures par caméra et au fait que même pour certaines méthodes commerciales l'utilisation de peinture ou de pastilles microbilles (pour le scanner auto positionnée Handyscan de la société CREAFORM) peut permettre d'avoir de meilleures conditions de mesure. Si l'objet ne peut être peint un changement des conditions de l'essai comme la nature du rayonnement peut être parfois une solution.

Le deuxième phénomène est l'existence de zones cachées qui compromet la réussite de cette stratégie. Ce problème est illustré sur la figure 1 . Il est possible d'y voir que les points B et $\mathrm{B}^{\prime}$ se projettent sur la même ligne de frange sur le capteur de l'appareil photographique. Ainsi, la présence d'une surface solide au point $\mathrm{B}^{\prime}$ peut faire apparaître deux raies lumineuses différentes comme une 


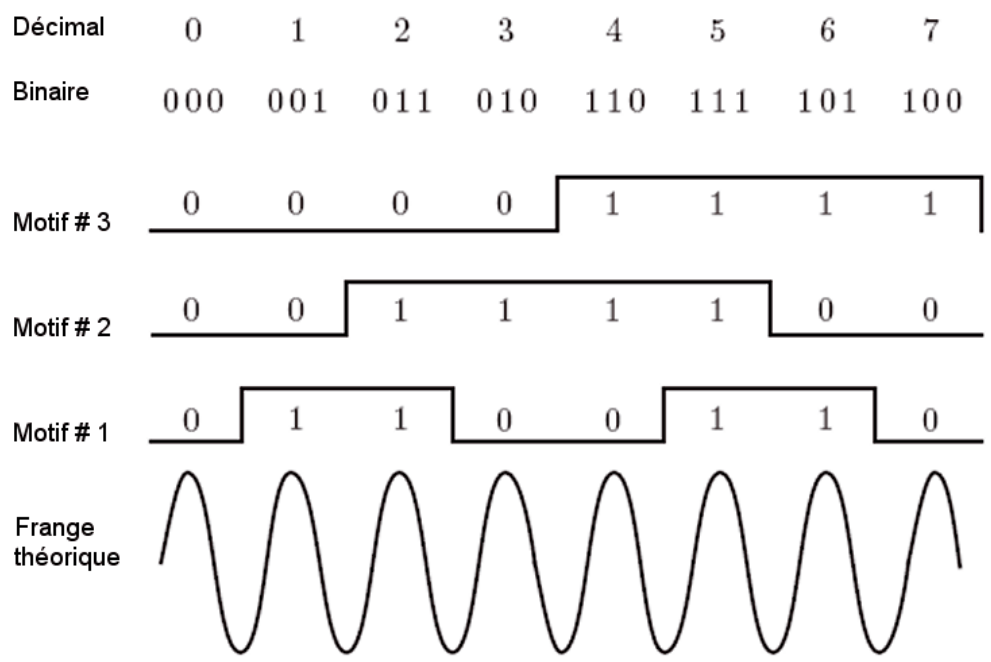

Fig. 3. Codage Gray de la phase du motif périodique. $N$ motifs permettent de coder $\left(2^{N}-1\right)$ positions. Le motif le plus fin a une période deux fois plus longue que le motif théorique.

seule ligne. L'examen local de la photographie ne peut permettre la distinction entre les deux franges comme l'illustre la petite photographie de la figure 1.

\subsection{Le codage Gray des franges}

Après ces réflexions sur les problèmes d'identifications de la phase, l'utilisation d'un codage de chaque fange par un code binaire Gray est proposée à l'étudiant. Cette solution limite la résolution spatiale mais résout le problème des zones cachées car la phase du motif est définie indépendamment de l'environnement du point de l'image ainsi considéré. Le principe de cette méthode est illustré sur la figure 3 .

Huit images sont projetées successivement sur l'objet. Manuellement ceci peut prendre une trentaine de secondes à deux personnes. L'une pour le projecteur et l'autre pour l'appareil photographique. En automatisant la procédure le temps nécessaire n'est plus qu'une ou deux secondes. Ces huit images permettent l'obtention de 255 franges virtuelles parfaitement identifiables à condition d'identifier les zones claires des zones pas éclairées. Cette condition est plus facile à satisfaire si le réseau le plus dense donne des bandes lumineuses de plusieurs pixels de large. Ainsi la détection peut se faire sur une petite surface de l'image.

\section{Extraction des données}

Afin de pouvoir extraire les données tridimensionnelles des images, il est nécessaire de définir un plan de référence dans lequel les franges sont non déformées et qui va servir de référence pour les mesures. Remarquons que l'activité pédagogique étant limité à $6 \mathrm{~h}$ la prise en compte des distorsions géométriques des images par le système optique de l'appareil photographique n'est pas prise en compte par l'étudiant. Une fois le plan de référence défini, les données tridimensionnelles sont alors extractibles avec une densité de points n'excédant pas le nombre de franges généré par le codage Gray.

Le travail de l'étudiant à ce niveau est double. Le travail initial est d'écrire une procédure simple sur un logiciel de traitement d'images. Cette macro-commande doit balayer automatiquement les $N$ images projetées sur l'objet pour recueillir sur un petit domaine surfacique l'intensité lumineuse. En tenant compte de la luminosité moyenne local un seuillage est alors effectué pour définir au mieux si les pixels mesurés correspondent à 1 ou à 0 . Le second travail est de définir la taille de la zone d'analyse et la distance entre deux positions successives.

Après conversion du code Gray en nombre décimal, l'étudiant obtient l'identification de la frange passant par le point dont les coordonnées $X O Y$ sont connues par la matrice de changement de repère. Remarquons que, de part la structure du code Gray, l'identification de franges aberrantes est possible, mais cela est rarement implémenté dans la procédure de traitement. Si la détection de la frange est correcte alors il ne reste plus qu'à comparer la position de la frange avec sa position théorique sur le plan de référence. La différence de position permet de calculer la distance entre le point considéré de la surface et le plan de référence. Ce calcul ne fait intervenir que des notions élémentaires de géométrie. Il peut donc faire facilement l'objet d'une estimation de l'erreur de mesure sous l'hypothèse que les aberrations optiques sont inexistantes ou corrigées. Les étudiants doivent également examiner le rôle des paramètres de l'expérience comme l'angle $\theta$ et les distances $D$ et $H$ (voir Fig. 1).

Après extraction des données géométriques contenues dans les images, il est possible d'importer les données dans une solution de CAO ou dans l'environnement d'un modeleur surfacique afin de reconstruire une surface par 

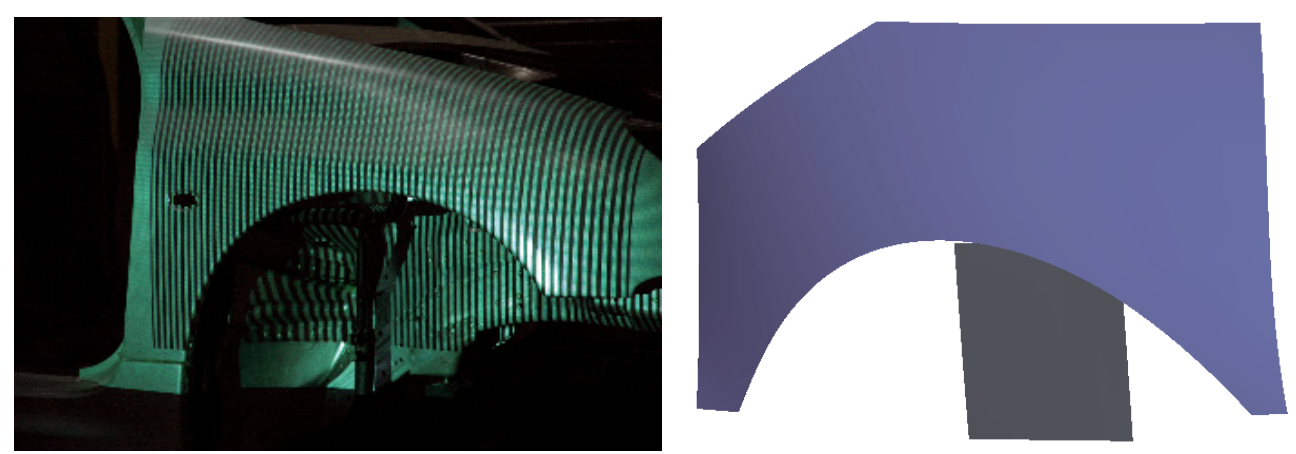

Fig. 4. Prise de vue lors de la mesure et début de reconstruction en faible densité de points.

lissage du nuage de points. Cette opération est visible sur figure 4. La possession d'un modèle numérique de référence permet alors la comparaison avec la reconstruction ainsi réalisée.

\section{Conclusions}

La mise en application de la mesure optique d'une pièce mécanique avec des moyens limités est possible comme le montre l'expérience décrite. Remarquons que des dispositifs encore plus simples furent utilisés par d'autres. Ainsi certains chercheurs proposèrent même de faire une mesure avec l'ombre d'une baguette balayant l'objet à mesurer.

L'approche proposée dans cet article permet aux étudiants de prendre conscience des limites et contraintes de ce type de méthode optique. C'est donc un dispositif pédagogique intéressant car il montre que les méthodes commerciales s'appuient sur des méthodes reproductibles à peu de frais même si la précision de ce dispositif reste limitée de part un étalonnage moins fiable. De plus, comme les traitements doivent être programmés par l'étudiant il peut plus facilement se rendre compte du perfectionnement de certains logiciels de traitement nécessaire à la mesure 3D par dispositif optique. 